\title{
Laboreal
}

Volume $7 \mathrm{~N}^{\circ} 2$ | 2011

Varia

\section{El error humano y la gestión de seguridad : la perspectiva sistémica en las obras de James Reason}

O erro humano e a gestão da segurança : uma perspectiva sistémica na obra de James Reason

L'erreur humaine et la gestion de la sécurité : l'approche systémique dans les œuvres de James Reason

The human error and the safety management: a systemic approach in the work of James Reason

\section{Mauro Marchitto}

\section{OpenEdition}

\section{Journals}

Edición electrónica

URL: http://journals.openedition.org/laboreal/7750

DOI: $10.4000 /$ laboreal. 7750

ISSN: 1646-5237

\section{Editor}

Universidade do Porto

\section{Referencia electrónica}

Mauro Marchitto, «El error humano y la gestión de seguridad : la perspectiva sistémica en las obras de James Reason », Laboreal [En línea], Volume 7 №2 | 2011, Publicado el 01 diciembre 2011

consultado el 10 diciembre 2020. URL : http://journals.openedition.org/laboreal/7750 ; DOI : https:// doi.org/10.4000/laboreal. 7750

Este documento fue generado automáticamente el 10 diciembre 2020.

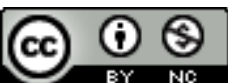

Laboreal está licenciado com uma Licença Creative Commons - Atribuição-NãoComercial 4.0 Internacional. 


\section{El error humano y la gestión de seguridad : la perspectiva sistémica en las obras de James Reason}

O erro humano e a gestão da segurança : uma perspectiva sistémica na obra de James Reason

L'erreur humaine et la gestion de la sécurité : l'approche systémique dans les cuuvres de James Reason

The human error and the safety management: a systemic approach in the work of James Reason

Mauro Marchitto

\section{NOTA DEL EDITOR}

Manuscrito recibido en : Junio/2011

Aceptado tras peritaje : Octubre/2011

Deseo agradecer a José Juan Cañas, Catedrático de Ergonomía Cognitiva de la Universidad de Granada (España) y mi personal mentor en estos últimos años. Sus sugerencias siempre me han indicado la mejor manera de impostar mis reflexiones. Deseo también agradecer a Jesús Villena, responsable del Editorial Modus Laborandi de Madrid, para las fructuosas conversaciones que tuvimos y que espero seguiremos teniendo, y para su importantísimo apoyo en la realización de este trabajo.

\section{Presentación}

1 En este artículo se presenta la obra de James Reason, Profesor de Psicología de la Universidad de Manchester, y experto reconocido a nivel mundial en el estudio del error humano en sistemas tecnológicos de alto riesgo y en el papel desempeñado por 
éste en los grandes desastres ocurridos en algunos dominios de producción industrial y de servicios. La producción científica del Profesor Reason es enorme, considerando también las aplicaciones prácticas que de sus estudios se han podido llevar a cabo en muchas organizaciones. En este artículo nos focalizamos en 3 libros que la Editorial Modus Laborandi (Madrid) ha traducido al castellano recientemente. Estas obras son : $<<$ El error humano >> (Titulo original: Human Error, 1990), <<La gestión de los grandes riesgos >> (Titulo original: Managing the risks of organizacional accidents, 1997), y por último <<La contribución humana >> (Titulo original : The human contribution, 2008). La traducción al castellano de estas obras tiene el valor añadido de poner la teorización acerca del error y de la gestión de la seguridad en sistemas socio-técnicos complejos de este autor fundamental a disposición de la comunidad <<hispanohablante>> mundial en dominios y contextos a donde tal vez no ha tenido todavía la oportunidad de llegar.

2 A lo largo de este artículo nos centraremos en los aspectos que, en nuestra opinión, son fundamentales sobre el error y la seguridad de sistemas y que han sido desarrollados por el autor a lo largo de sus tres obras, intentando evidenciar como los mismos conceptos, modelos, y métodos de investigación y gestión de la seguridad han evolucionado tras años de estudio meticuloso de algunos dominios profesionales. Entre estos aspectos, cabe destacar por ejemplo el progresivo <<abandono >> de la perspectiva individual a la hora de estudiar el error o la prestación errónea en su efectivo contexto de ocurrencia, a beneficio de una perspectiva organizacional y sistémica, que distingue entre fallo activo (inmediatamente visible), y condición latente como un importante factor que contribuye a las evoluciones de algunas situaciones hacia el accidente. En otras palabras, la evolución podría expresarse como el paso de una visión de las personas como <<propensas al error>> a la de acción humana de por sí variable, que termina con ser incorrecta si se lleva a cabo en contextos propensos al error. Otro aspecto clave que hemos querido destacar es la atención dedicada en el último de esos libros a las recuperaciones heroicas, entendiendo entonces la ejecución correcta e incorrecta como dos caras de la misma moneda, la de la actuación humana.

3 El trabajo se articula en una primera sección en la que se reflexiona sobre el cambio de perspectiva (de personal a sistémica) que el mismo Reason ha adoptado a lo largo de su obra, contribuyendo mucho a que ese cambio se produjera también en seno a muchas organizaciones. La reflexión se articula también con subsecciones que tratan además de la evolución del «<Modelo del queso suizo»> (Swiss Cheese Model, SCM) y la consideración de la conducta correcta. La sección siguiente trata de la gestión de la seguridad, parte fundamental para cualquier intento de aplicación práctica de los conocimientos teóricos desarrollados, tanto para los análisis retrospectivos de incidentes como para los análisis prospectivos de seguridad.

\section{Desde el $<<$ error humano > a la $<<$ contribución humana >}

4 Si miramos a la evolución histórica de los sistemas industriales, de la tecnología, y de la automatización, podemos ver como en la tecnología se ha sustituido al operador humano en muchísimas actividades, trasformando dicho operador muchas veces en <<monitorizador >> de procesos automáticos. La reducción de errores $<<$ de ejecución»> en muchos sitios ha sido sustituida inevitablemente por un aumento de los errores de evaluación, de previsión, y de mantenimiento de los sistemas automáticos, con el 
resultado de que el <<Factor Humano»>, sigue siendo señalado como el responsable del $70 \%$ de los accidentes, un numero citado miles de veces. Sin embargo, la perspectiva optimista de los partidarios de la automatización <<fuertes>, que persigue la eliminación de los errores humanos por medio de una automatización completa de muchas operaciones, está lejos de realizarse, aunque los progresos tecnológicos sean efectivamente sensacionales. Las razones residen, muy probablemente, en el hecho de que no es tanto el nivel de automatización de un sistema el que determina la presencia/ ausencia de conductas erróneas o incorrectas, sino el conjunto de factores que afectan y regulan la ejecución humana en determinados sistemas: estos factores están relacionados con la organización, su cultura, sus políticas, sus procedimientos, y sus prácticas. El enfoque de la ingeniería (y de las técnicas de análisis de la conducta humana inventados por esa disciplina, como por ejemplo las técnicas para el análisis de fiabilidad humana), es demasiado estricto (y hoy en día superado) para estudiar la acción humana en contextos operativos. Los seres humanos somos intrínsecamente creativos, adaptativos, y muchas veces heurísticos. En una palabra, variables. Por la misma razón, para abordar la gestión de la seguridad en sistemas complejos ya no es posible adoptar un modelo exclusivamente personal, enfocado sobre las características <<generales >> del ser humano estudiado en los laboratorios de las Facultades de Psicología y los errores típicos de su mente falible. Más bien, un modelo sistémico ve a la actuación humana como correcta o incorrecta en función del contexto en la que ocurre (y aceptable o inaceptable en función de las reglas escritas y que determinan la aceptabilidad de dicha acción) por los factores relacionados a la organización en sí, y por los objetivos globales y locales que en ésta se definen.

5 En la obra de Reason es posible vislumbrar una trayectoria parecida, yendo de un enfoque personal a uno sistémico. El enfoque de $<$ El error humanos> estuvo muy influenciado por la Psicología Cognitiva y por la necesidad de abordar el fenómeno del fallo de la conducta antes de todo definiendo una taxonomía que ayude a identificar e interpretar el error mismo. De hecho, ese primer libro fue principalmente pensado para los pares de Reason: los académicos de Psicología Cognitiva. La metáfora del ser humano como procesador de información simbólica transformada en planes de acción y consecuente ejecución era tan dominante en ese momento que no habría podido dejar de guiar la creación de la taxonomía. De hecho, la contribución de Rasmussen (1983) a la clasificación de los niveles de actuación humana se completa en este sentido con una clasificación de la actuación errónea, concentrándose en el proceso mental entendido como causa de dicha actuación. La dificultad empírica aparentemente insuperable permanecía, pero se abría la posibilidad de capturar los procesos mentales al nivel de investigación, sobre todo en un contexto real donde no se tiene el control típico de los contrastes de hipótesis de los experimentos de laboratorio. En un contexto operativo (por ejemplo una sala de control), solo es posible observar la actuación humana pero no su planificación mental o los pasos de un razonamiento. Y además, el propio contexto será el elemento fundamental que dará sentido a una actuación concreta y no a otra. De toda forma, a parte el intento de ir más allá de la sola clasificación del error (su descripción), es notable el propósito de estudiar las causas de un error (su explicación), y no solo su frecuencia de ocurrencia. Aunque desde esta perspectiva se sigue quizás todavía atado a la concepción de un ser humano falible de por sí, debido a los limitados recursos y capacidades mentales. 
6 En las conceptualizaciones posteriores, Reason empieza, en mi opinión, a dejar la perspectiva demasiado estricta de la Psicología académica para contribuir en manera fundamental e irrepetible a la Ergonomía. El nacimiento de los primeros modelos organizativos relacionados con el error humano, la inclusión de los gestores en las causas de los accidentes, la conceptualización de un contexto como propenso al error o a la infracción, son todos puntos que expresan la asunción básica de que aunque la mente humana es falible, el tema fundamental es averiguar dónde y cómo pueda fallar, para poder soportarla en consecuencias negativas que la limitan. En otras palabras, en las realidades industriales y de producción de servicios, hay que enfocarse en los lugares de trabajo, las condiciones en las que se opera, y los elementos de gestión que promueven la falibilidad humana.

7 Este último punto es la consecuencia empírica de una distinción muy importante en los análisis de incidentes, entre lo que es propiamente una causa y lo que es una condición (latente). Los fallos activos son desencadenantes locales que juntos a unas precondiciones pueden dar origen a un accidente. Las condiciones latentes son universales y comunes a virtualmente todas las organizaciones, mientras que los fallos activos marcan las diferencias entre ellas. Esta distinción es una contribución importante aportada a la metodología de investigación en sí, pero también a la propia filosofía de los estudios retrospectivos con objetivos explicativos : de hecho orienta la búsqueda a las condiciones contextuales y sugiere contemporáneamente la despersonalización del error activo (el de <<primera línea>>), lo cual hubiera podido ocurrir a otro operador en el mismo contexto.

8 La problemática fundamental a nivel de gestión no solo de la seguridad, sino del negocio en general, acaba con ser necesariamente la monitorización de estas condiciones latentes y su rectificación con medidas de procesos proactivas, no solo reactivas.

9 Si en $<<$ El error humano >> estas consideraciones toman cuerpo en la parte final del libro, en $<<$ La gestión de los grandes riesgos $>>$ representan el corazón de toda la obra, la cual hace hincapié en el modelo sistémico tanto para edificar la teoría sobre los accidentes organizacionales, como para definir métodos de gestión de seguridad proactivos. Por esta razón podemos decir que el cambio de perspectiva que acabamos de mencionar se resume muy bien relacionando el título del libro del 1990 al de 2008, tal como se ha hecho en el título de esta sección.

10 A continuación, destacamos unos puntos esenciales que han contribuido a la definición de un enfoque sistémico en relación a la gestión de seguridad.

\section{La perspectiva sistémica : contextos propensos al error y a las infracciones}

11 En "La Contribución Humana", el Capítulo 4 sobre infracciones (cuya importancia está relacionada también con las diversas maneras de ver los actos peligrosos, como se expone en el Capítulo 5), representa una importante profundización, respeto a cuanto ya estaba esbozado en el libro anterior: el esquema de las infracciones (correctas o incorrectas, gratificantes o ingratas) en presencia de normas buenas, malas, o en ausencia de normas da lugar a 12 tipos de situaciones diferentes que constituyen, todas 
juntas, un modelo descriptivo y al mismo tiempo una herramienta interpretativa de un especifico contexto operacional.

Es un valor añadido el hecho de que se llegue a hablar de acción correcta/incorrecta, porque eso quiere decir que se ata la actuación humana al contexto en el que ésta ocurre, considerando su exactitud o no en función de los objetivos que en ese contexto se trata de alcanzar. De la misma manera, distinguir entre acciones gratificantes y no gratificantes significa añadir a la conceptualización de las infracciones la dimensión hedonística individual, en la cual muchas veces residen las causas de maneras de actuar tal vez en contra de las reglas, pero que son mucho más satisfactorias, considerando la difícil aplicabilidad de una regla mal redactada (como se ha visto en muchos casos, y en diferentes dominios).

Las taxonomías no serían útiles si no ayudaran a interpretar correctamente la realidad $\mathrm{y}$, en una cierta medida, a poder predecirla. Un esquema interpretativo de las infracciones va a completar el discurso sobre el error. Tal como el error es en parte determinado por factores locales <<latentes〉>, también las infracciones deliberadas pueden ser fomentadas (y aceptadas) por el propio contexto, en función también de los tratos culturales de una organización dada.

Hablar de error humano sin dotarse de esquemas, modelos y herramientas que ayuden a reconocerlo en los contextos operativos, y sin considerar qué elementos específicos de ese contexto habrán afectado el normal desempeño de una actividad, significa satisfacer solo necesidades clasificatorias y puramente teóricas que no sirven de mucho a la ergonomía y a la psicología del problema.

\section{El modelo del queso suizo (Swiss Cheese Model, SCM)}

El célebre Modelo del Queso Suizo (Swiss Cheese Model, SCM) es un modelo identificativo de toda la obra de James Reason, aunque como él mismo ha declarado muchas veces, no fue él el autor de tan afortunado apodo. Lo que es importante es caer en la cuenta de que este modelo representa claramente la necesidad de adoptar un modelo sistémico a la vez de abordar la seguridad organizacional y las investigaciones de eventos incidentales. En años recientes, el modelo ha sido revisitado por el propio autor y otros eminentes estudiosos de seguridad en sistemas de alto riesgo (Reason, Hollnagel, \& Paries, 2006) : se ha reconocido su débil poder predictivo es decir, la capacidad que tiene el SCM de hacer predicciones altamente probables pero poco precisas. Sin embargo, aunque su poder como base para hacer previsiones y análisis de eventos es escasa en comparación con otros enfoques o modelos, hay que evidenciar como el SCM haya sido uno de los instrumentos pedagógicos más poderoso para vehicular en los dominios industriales el modelo sistémico en la gestión de seguridad. Curiosamente, como se ha hecho evidente, parece que la única persona que ha avanzado críticas hacia el modelo en los últimos años ha sido a menudo el propio autor, al considerar la ubicuidad del SCM en la literatura mundial sobre la gestión de la seguridad.

Ya desde sus primeras versiones (Capitulo $7 \mathrm{de}<<$ El error humano $>>$ ), el futuro modelo <<del queso suizo > mostró el gran valor de introducir los factores latentes en la representación de un sistema socio-técnico complejo, considerando todos los niveles estructurales que lo componen, dirección de empresa incluida. Este hito representó un paso necesario y, para aquellos tiempos, incluso valiente. La necesidad de superar una 
cultura exclusivamente tecno-formativa, es decir que solo confía en un diseño técnico seguro y "a prueba de error" junto a reglas operativas fiables para la gestión de los procesos, quedaba clara a la luz de los importantes, trágicos, acontecimientos en varios dominios industriales. Desastres como el de Chernobyl, del Challenger, de Bhopal y de Three Miles Island, (y las sucesivas investigaciones) revelaron como el axioma implícito en seno a muchas empresas, que definía la seguridad como la suma de una buena ingeniería, una tecnología fiable y unos buenos procedimientos, era antes de todos peligroso, por el hecho de ignorar la componente humana de gestión y las habilidades de rescate del ser humano con respecto a la máquina, a parte de una falta de investigación sobre las condiciones (latentes) en las cuales las acciones de los operadores se llevaban a cabo. Con este modelo empieza a cambiar la visión de la gestión de la seguridad, requiriendo a las organizaciones que se enfoquen sobre ellas mismas a nivel sistémico a la vez que buscan datos y los transforman en informaciones significativas sobre el estado de salud de la empresa o, siguiendo la metáfora de James Reason, sobre donde <<se sitúa el barco en el espacio de navegación>>.

En sus sucesivas formulaciones se evidencia aún más como los factores contextuales, juntos a las decisiones equivocadas a nivel direccional (afectadas por los rasgos culturales de una organización), favorezcan el error, alimentando entonces un contexto <<propenso>> a éste. Toda la reflexión sobre la génesis del error según esta perspectiva viene relacionada explícitamente con la dinámica incidental y, por ende, con los verdaderos factores contribuyentes de los desastres. Los fallos visibles de los operadores de primera línea son el elemento visible, pero consecuencial, de unas concausas cuyo origen puede remontarse en el tiempo y en el espacio (por ejemplo en las reuniones de planificación de la producción).

En paralelo, se incluye en el marco teórico (recordando su fuerte finalidad práctica) el tema importantísimo de las infracciones y la necesidad de su explicación antes de su represión: la existencia de infracciones correctas, de eventuales cumplimientos erróneos, o incluso de casos de improvisación, son matices que podemos comprender muy bien gracias al esquema interpretativo citado antes. Bajo esta perspectiva, la presencia de malas normas o en su lugar la ausencia de normas acerca de unas situaciones posibles se relaciona con los fallos a nivel de programación de los procesos o con un vacío formativo que contribuyen a un contexto propenso a la infracción.

19 En la mayoría de los casos, entonces, la ocurrencia de un desastre no puede ser atribuible a la sola primera línea, a los fallos visibles de pilotos, operadores, cirujanos, comandantes de barcos, etc. Más bien, estos errores activos son en muchas ocasiones el resultado de la normal variabilidad de la actuación humana, aunque sea en un contexto fuertemente reglamentado por unos procedimientos operacionales, y en gran medida determinado por las decisiones de gestión. Como consecuencia, solo el estudio en el que se adopta una perspectiva sistémica puede ser la mejor aproximación a la vez que se adoptan las medidas proactivas de seguridad.

20 A esta conceptualización contribuyeron las investigaciones de accidentes significativos fundamentales para que se pusiese en marcha un proceso necesario de cambio cultural, como el informe Mahon y el informe Moshansky, tal como se evidencia en $<$ La contribución humana>> (Parte III). A lo largo de toda su obra, Reason expresa repetitivamente la necesidad de un cambio cultural en la manera de entender la gestión de seguridad hacia una perspectiva sistémica. En muchos ámbitos esta visión ha sido adoptada e incluida, por ejemplo, en los programas de formación desde hace mucho 
tiempo. Aunque, a veces, se ha tardado bastante tiempo en trasladarla a las diferentes áreas de un sistema socio-técnico en cuestión. En la aviación comercial, por ejemplo, la formación del personal de vuelo en contenidos que no fuesen solo técnicos y de operación, sino también relacionados a los Factores Humanos, la Psicología Social, la comunicación y el trabajo en equipo, han sido parte integrante de la formación orientada a la gestión de los recursos de tripulación (Crew Resources Management, CRM) desde los años '70. Aunque, lo que sí se ha tardado en hacer ha sido extender este tipo de formación al personal responsable del control aéreo y, solo recientemente a los técnicos de mantenimientos.

\section{La ejecución correcta y las recuperaciones heroicas}

21 Un elemento novedoso del libro $<<$ La contribución humana $>$ es todo el discurso sobre las recuperaciones heroicas. Podríamos afirmar que toda esta obra gravita alrededor de este concepto clave: los errores y las ejecuciones correctas son dos aspectos complementarios de la actuación humana. Tras un enorme esfuerzo orientado a comprender los accidentes, los errores que a ellos contribuyen, las causas de los mismos a varios niveles de una organización considerando contexto de ocurrencia (y la cultura que en buena medida lo determina), llega un momento en que cabe preguntarse por qué cuando las cosas van bien, van bien. Y sobre todo, porque cuando las cosas van mal, la contribución humana puede ser el elemento fundamental para recuperar una situación diversamente sin salida. Como se argumenta a lo largo del libro y con ejemplos iluminantes sacados de diferentes dominios $\mathrm{y}$, con el valor añadido de la reconstrucción histórica, las cuestiones sistémicas son tan importantes para las recuperaciones heroicas como lo son para la inducción de actos peligrosos. Por supuesto, el efecto conjunto de algunas características personales es el elemento necesario para que ocurra una recuperación heroica. El caso es que estas capacidades son en gran parte fruto de experiencia operativa, conocimiento (conciencia) de los peligros que amenazan las operaciones y competencia, todos factores que se desarrollan en el propio contexto operativo : no son regalos de la providencia.

El ser humano es el <<eslabón débil > de la cadena operacional porque comete errores pero al mismo tiempo su versatilidad, sus capacidades de razonamiento heurístico y su creatividad lo hacen el recurso más potente para recuperar situaciones límite o ya trágicas. En este libro es evidente la visión positiva del ser humano que ya no viene a ser visto como inevitablemente falible e imperfecto, sino más bien como variable en su contexto, incluso a la vez de operar en sistemas altamente reglamentados. Esta evolución de perspectiva, en una cierta medida, pone en cuestión el mismo concepto de error : la contribución humana no es errónea, más bien será correcta o incorrecta en función de los objetivos locales y globales. El contexto de actuación, en cuanto elemento inseparable de la actividad humana afectará al resultado de dicha actividad y los medios adoptados para su alcance (lícitos y no). La consideración del ser humano como <el anillo fuertes> de cualquier proceso laboral viene a ser un supuesto irrenunciable de cualquier sistema de gestión de la seguridad y un rasgo cultural necesario en la gestión de las actividades de alto riesgo. 


\section{La gestión de la seguridad y las bases de la prevención} incorrecta, no ocurre <<fuera > de su contexto. Una taxonomía de los errores es un instrumento útil para interpretar unos eventos y generar conocimiento sobre éstos. Pero no hay que cometer el error de considerar <<explicada $>$ una conducta porque una taxonomía sugiere esa interpretación. Para remontarnos a las causas de unos eventos hay que considerar la organización en la que ocurrieron en su complejidad y dinámicas de proceso. El nivel de comprensión y de <<auto-análisis > de una organización será mejor cuanto más disponibilidad haya de información significativa y recursos y dedicación para investigarla. En pocas palabras, será la específica gestión de la seguridad la que determinará la duración del negocio en las actividades de alto riesgo y no tanto la bondad de los instrumentos investigativos de por sí.

Lo que Reason nos va explicando es que un equilibrio óptimo entre la adopción de métodos de análisis de eventos y la puesta en marcha de medidas de procesos proactivas es la clave fundamental de la gestión de la seguridad: saber interpretar lo que ocurre, poder hacer unas previsiones (que no equivale a establecer un objetivo de producción) y evaluar cuanto se ha investigado son los hitos de un buen sistema de gestión de la seguridad. Queda claro que estos procesos nunca alcanzan un estado final : no hay ninguna <<llegada >> en la gestión de la seguridad, solo un <<largo viaje >>.

En el Capítulo 6 de $<<$ La gestión de los grandes riesgos >> se propone la metáfora del <<espacio de seguridad >> como de un espacio de navegación en el que la organización se mueve aprovechándose de unas cuantas herramientas para navegar hacia el extremo de creciente resistencia, más que de creciente vulnerabilidad. De aquí la visión positiva de la seguridad y su definición como de un proceso de implementación activo y no como el simple registro de eventos adversos. La ausencia de accidentes no hace una organización segura de por sí, sino, a lo mejor, bastante afortunada. La ausencia de programas activos de recogida de informaciones relevantes detiene la atención hacia los <<patógenos residentes>>, es decir las condiciones latentes, y acerca la organización más a un "barco indemne", una posición intimadamente peligrosa.

Una parte fundamental del libro <<La gestión de los grandes riesgos >> viene entonces a ser aquella en la que se exponen unos métodos para le gestión del error (un conjunto de <<ayudas a la navegación>> en el espacio de seguridad) y el análisis de seguridad. La revisión de algunas de estas herramientas evidencia los principios desarrollados anteriormente que definen la seguridad como un esfuerzo activo en la continua implementación de apropiadas medidas de proceso a todos los niveles de la organización.

Un aspecto fundamental del éxito de estos métodos de gestión de seguridad ha sido el hecho de que las informaciones significativas son recogidas por los operadores (de primera línea y de mantenimiento), que vienen a ser parte activa del proceso de análisis. Para que los propios operadores sean observadores fiables y motivados, estas herramientas tienen que basarse en aspectos medibles y bien conocidos, para los cuales los operadores internos a la organización son más idóneos que un equipo de <<expertos»> extraños al propio contexto de trabajo. La medición de los tipos genéricos de fallo (General Failure Type, GFT) opera a un nivel que no mira a corregir las acciones 
individuales, sino a detectar patrones de procesos más generales, responsables de las condiciones en las cuales los errores activos son casi inevitables. Los actos peligrosos no se pueden conocer de antemano. Los tipos genéricos de fallo (relacionados con hardware, diseño, mantenimiento, comunicaciones, procedimientos, organización, etc.) sólo se pueden conocer de antemano implementando apropiados métodos de recogidas de datos relevantes y transformándolos en información saliente. Es esta la «<novedad > más importante respeto al enfoque <<tradicional $>$ que se preocupaba de implementar medidas correctivas de la conducta individual más que enfocadas a modificar las condiciones locales que pueden promover los errores. La tercera dimensión del modelo TRIPOD (la <<pata>> superior) representa la esfera de acción del modelo a un nivel más alto de lo de la conducta individual. El objetivo es cambiar al contexto más que la conducta humana.

Es una filosofía de gestión de la seguridad que intenta ser cuanto más proactiva en vez que solo reactiva, recogiendo datos acerca de aspectos medibles y observables que sirven de indicador sobre la magnitud de tipos genéricos de fallos (las condiciones latentes). Es esta la parte susceptible de cambio, y no los "estados mentales fugaces". La Psicología Cognitiva, a pesar de haber dado a lo largo de su desarrollo un conspicuo número de modelos, explicaciones, esquemas e interpretaciones de los procesos mentales humanos y de los errores a ellos más típicamente asociados, se relaciona a menudo con aspectos no directamente observables, difícilmente medibles, y con escasa probabilidad de ser objeto de medidas correctoras. Los fallos activos debidos a un específico proceso mental, pueden contribuir a un accidente solo si ocurren en unas condiciones locales que impiden la recuperación de estos fallos. Estas condiciones sí que pueden ser conocidas y mejoradas con medidas que fortalecen el sistema de defensas en profundidad. Por ende, un accidente organizacional se evita conociendo lo más fielmente posible los factores locales que pueden desempeñar un papel en generar en dichos accidentes, y que tal vez no son estrictamente relacionados con la producción. Todos estos factores no son posibles de ser arreglados con un montón de procedimientos individuales orientados solo a la seguridad personal y no del sistema. En <<La gestión de los grandes riesgos >> cabe destacar también la discusión acerca de la posición de los entes reguladores de dominios a alto riesgo: el autor delinea, tanto desde una perspectiva histórica como desde una más pragmática de gestión, las contribuciones para fomentar una regulación con medidas de sistema y ya no más solo orientadas a la corrección de la actuación individual (Capitulo 8).

29 Es esta otra contribución fundamental en ese libro para la ampliación de la perspectiva sistémica.

\section{Conclusión}

La publicación en castellano de las tres principales obras de James Reason representa una oportunidad de lectura irrenunciable, tanto para los estudiantes de Ergonomía (y de las disciplinas que participan a la investigación ergonómica como por ejemplo la Psicología, la Ingeniería y la Sociología del trabajo), como para los profesionales y los <<prevencionistas >> que no hayan conocido estos textos en su versión original.

31 Si en $<<$ El error humano $>$ se nota mucho la influencia de la formación académica (en Psicología Cognitiva) del autor a la vez de proponer taxonomías clasificatorias del error humano, en las obras sucesivas esta visión estrictamente relacionada con el 
procesamiento humano de la información deja el paso a la adopción de una perspectiva sistémica para poder estudiar la actuación humana en reales contextos de trabajo a alta peligrosidad. El estudio de las causas de grandes accidentes, esbozado en $<<$ El error humano $>>$ y profundizado en $<<$ La gestión de los grandes riesgos $>>$, ha sugerido al autor no considerar sólo los proceso mentales de los operadores de primera línea sino también las condiciones locales en las que la actividad humana se realiza. La gestión de seguridad en sistemas socio-técnicos complejos (de producción industrial y de servicios) no puede prescindir, bajo esta perspectiva, la implementación de medidas proactivas de procesos tras la monitorización continua de áreas críticas que pueden alimentar contextos propensos a los errores y a las infracciones (los $<<$ tipos genéricos de fallo>> del método TRIPOD).

En $<<$ La contribución humana > el modelo sistémico adquiere ulterior importancia por ser también la clave de lectura de la ejecución correcta y sobre todo, de las recuperaciones heroicas : aunque parezca que las habilidades personales (junto a una considerable dosis de buena suerte) sean los únicos responsables de las recuperaciones heroicas, aparece claro como estas mismas habilidades sean fruto del compromiso, del conocimiento, y de unas actitudes a pensar en los peligros como probables y entonces posibles, elementos estos fomentados por el propio contexto de trabajo.

La lectura de estos tres libros es un paso en mi opinión fundamental para todos los profesionales y los gestores de dominios críticos para la seguridad, no solo para los varios empleados del departamento de seguridad de una empresa. En este trabajo de recensión solo he querido destacar unos conceptos en mi opinión centrales para poner en evidencia la evolución de la perspectiva sistémica a lo largo de los 3 libros : entre estos figuran el modelo del <<queso suizo>>, la interpretación de las infracciones y la gestión preventiva de la seguridad.

\section{BIBLIOGRAFÍA}

Mahon, P., Mr. Justice (1981). Report of the Royal Commission into the Crash on Mount Erebus, Antarctica, of a DC-10 Aircraft Operated by Air New Zealand Limited. Wellington: New Zealand.

Moshansky, V. P., Mr. Justice (1992). Commission of inquiry into the Air Ontario crash at Dryden, Ontario. Final Report. Ottawa: Ministry of Supply and Services.

Rasmussen, J., (1983). Skills, rules, knowledge: signals, signs and symbols and other distinctions in human performance models. IEEE Transactions: Systems, Man \& Cybernetics, SMC-13, 257-267.

Reason, J. (2008). El error humano. Madrid : Modus Laborandi. [Edición original : Human Error. Nueva York : Cambridge University Press, 1990.]

Reason, J. (2010). La gestión de los grandes riesgos. Principios humanos y organizativos de la seguridad. Madrid: Modus Laborandi. [Edición original: Managing the Risks of Organizational Accidents. Aldershot : Ashgate, 1997.] 
Reason, J. (2011). La contribución humana. Actos peligrosos y acciones ejemplares. Madrid: Modus Laborandi. [Edición original: The Human Contribution. Unsafe Acts, Accidents and Heroic Recoveries. Aldershot: Ashgate, 2008.]

Reason, J., Hollnagel, E., \& Paries, J. (2006). Revisiting the $<<$ Swiss Cheese $>>$ Model of Accidents. EUROCONTROL, EEC Note No. 13/06. Brétigny-sur-Orge : EUROCONTROL Experimental Centre.

\section{RESÚMENES}

Este articulo revisa brevemente tres libros de James Reason (<<El error humano>>, <<La gestión de los grandes riesgos >>, y <<La contribución humana>>, más precisamente), eminente experto a nivel mundial de error humano y ergonomía organizacional. A través de estos libros es posible entender como la gestión de la seguridad en los sistemas socio-técnicos complejos haya requerido un cambio de perspectiva en las últimas décadas, tanto a nivel cultural como a nivel de las prácticas de gestión de seguridad, sobre todo después de importantes, trágicos accidentes. Se puede entender este cambio fundamental si se mira por ejemplo a la difusión de modelos <<organizativos >> de los incidentes (que miran a explicarlos sin abarcar solamente el personal de <<primera línea>>), a la adopción de una perspectiva sistémica también de cara a las medidas preventivas de gestión de seguridad, o a las consideraciones de las condiciones latentes que promueven los inevitables fallos activos en los contextos operacionales. Estos aspectos son brevemente discutidos aquí, reenviando el lector a la lectura integral de las obras señaladas, ahora traducidas al castellano y publicadas por la editorial Modus Laborandi de Madrid.

Este artigo revê brevemente três livros de James Reason $(<<0$ erro humano $><<$ A gestão de grandes riscos $>\mathrm{e}<<\mathrm{A}$ contribuição humana $>$, para ser preciso), um especialista a nível mundial em erro humano e ergonomia organizacional. Através destes livros é possível compreender como a gestão da segurança nos sistemas sócio-técnicos complexos exigiu uma mudança de perspectiva nas últimas décadas, tanto culturalmente como em termos de práticas de gestão de segurança, especialmente depois da ocorrência de acidentes importantes e trágicos. Podemos entender esta mudança fundamental se olharmos, por exemplo, para a disseminação de modelos <<organizacionais>> dos incidentes (que parecem explicá-los sem abarcar unicamente o pessoal de "primeira linha"), para a adopção de uma perspectiva sistémica também relativamente às medidas preventivas de gestão da segurança, ou para a consideração das condições latentes que promovem as inevitáveis falhas activas nos contextos operacionais. Estes aspectos são brevemente discutidos aqui, reenviando o leitor para a leitura integral das obras mencionadas, agora traduzidas para castelhano e publicadas pela editora Modus Laborandi de Madrid.

Cet article propose une présentation de trois livres de James Reason - L'erreur humaine, Managing the risks of organizational accidents, The human contribution -, expert reconnu au niveau international sur l'erreur humaine et l'ergonomie organisationnelle. Au travers de ces œuvres on comprend comment la gestion de la sécurité, dans les systèmes socio-techniques complexes, a nécessité une évolution en termes d'approche ces dernières décennies, aussi bien au niveau culturel que dans les pratiques de gestion des risques, surtout suite à de grandes catastrophes et accidents importants. On peut comprendre ce changement fondamental si l'on regarde par exemple, la diffusion de modèles sur l'organisation pour l'analyse d'évènements (qui cherchent à expliquer ceux-ci en allant au-delà des opérateurs qui se retrouvent en première ligne), jusqu'à l'adoption d'une approche systémique intégrant la prévention et la gestion des risques, ainsi que la prise en compte des défaillances latentes contribuant aux inévitables erreurs actives dans les contextes opérationnels. Ces aspects sont discutés brièvement et on invite le 
lecteur à se référer aux trois œuvres en question traduites à l'espagnol par les Editions Modus Laborandi.

This paper briefly revises three books written by James Reason (namely $<<$ Human Error $>$, <<Managing the risks of organizational accidents >>, and $<<$ The human contribution >>), a worldwide eminent expert of human error and organizational ergonomics. Through these books it is possible to understand how safety management in high risk socio-technical systems required a perspective shift in the last decades, concerning both safety culture and safety management practices, especially after enormous, tragic catastrophes. This fundamental change can be comprehended thanks to several important aspects: among them, the spreading of <<organizational >> models of accidents (which try to explain errors by focusing not only on first line operators), the adoption of a systemic perspective also in relation to preventive measures of safety management, and the consideration of latent conditions able to promote the inevitable active errors in operational contexts. All these aspects are discussed here, inviting the reader to the integral reading of the books reviewed, now translated to Spanish and published by Modus Laborandi, Madrid.

\section{ÍNDICE}

Mots-clés: erreur humaine, défaillance latente, violations, gestion de la sécurité, perspective systémique

Palabras claves: error humano, condición latente, infracciones, gestión de seguridad, perspectiva sistémica

Keywords: human error, latent condition, violations, safety management, systemic approach Palavras-chave: erro humano, condição latente, infracções, gestão da segurança, perspectiva sistémica

\section{AUTOR}

\section{MAURO MARCHITTO}

Grupo de Ergonomía Cognitiva, Departamento de Psicología Experimental, Facultad de Psicología, Universidad de Granada, Campus Cartuxa s/n, 18071, Granada, España

maurom@ugr.es 